\title{
Patterns of Autobiographical Memory Loss in Medial-Temporal Lobe Amnesic Patients
}

\author{
R. Shayna Rosenbaum ${ }^{1,2}$, Morris Moscovitch ${ }^{2,3}$, Jonathan K. Foster ${ }^{4}$, \\ David M. Schnyer ${ }^{5}$, Fuqiang Gao ${ }^{6}$, Natasha Kovacevic ${ }^{2}$, \\ Mieke Verfaellie $^{7}$, Sandra E. Black ${ }^{2,3,6}$, and Brian Levine ${ }^{2,3}$
}

\begin{abstract}
The issue of whether the hippocampus and related structures in the medial-temporal lobe (MTL) play a temporary or permanent role in autobiographical episodic memory remains unresolved. One long-standing belief is that autobiographical memory (AM), like semantic memory, is initially dependent on the MTL but ultimately can be retained and recovered independently of it. However, evidence that hippocampal amnesia results in severe loss of episodic memory for a lifetime of personally experienced events suggests otherwise. To
\end{abstract}

\section{INTRODUCTION}

A large body of evidence from studies on anterograde memory implicates the hippocampus (hippocampus proper, dentate gyrus, and subiculum) and related medial-temporal lobe (MTL) structures (entorhinal, perirhinal, and parahippocampal cortices) in the acquisition and short-term retention of explicit or declarative information. However, the specific types of memories affected by MTL $^{1}$ damage and the changes in brain organization that occur as memories age have been the subject of considerable debate. Recent attempts by several laboratories at characterizing remote (retrograde) memory loss for detailed personal episodes and for more general semantic facts in patients with MTL damage have produced conflicting results. The reason for the discrepancies across studies is that a variety of methods were used to measure autobiographical memory (AM) loss in patients who differed in terms of location and extent of damage (Moscovitch, Nadel, Winocur, Gilboa, \& Rosenbaum, 2006). The current study investigates remote AM in people with severe amnesia result-

\footnotetext{
${ }^{1}$ York University, Toronto, Ontario, Canada, ${ }^{2}$ Baycrest Centre for Geriatric Care, Toronto, Ontario, Canada, ${ }^{3}$ University of Toronto, Toronto, Ontario, Canada, ${ }^{4}$ Edith Cowan University, University of Western Australia \& Neurosciences Unit, Health Department of Western Australia, Perth, Australia, ${ }^{5}$ University of Texas at Austin, Texas, ${ }^{6}$ Sunnybrook Health Sciences Centre, Toronto, Ontario, Canada, ${ }^{7}$ Boston VA Healthcare System and Boston University School of Medicine, Boston, MA
}

test the opposing views, we conducted detailed investigations of autobiographical episodic memory in people with amnesia resulting from MTL lesions of varying extent. By combining precise quantification of MTL and neocortical volumes with sensitive measures of recollection of one's personal past, we show that the severity of episodic, but not semantic, AM loss is best accounted for by the degree of hippocampal damage and less likely related to additional neocortical compromise.

ing from MTL damage using a measure that is sensitive in detecting the capacity to re-experience personal episodic details and in differentiating them from semantic details (Levine, Svoboda, Hay, Winocur, \& Moscovitch, 2002). In addition, volumetric analysis within different regions of the MTL and the neocortex was used to determine the relation between patterns of MTL and neocortical damage and AM performance.

A number of theories have been advanced to account for findings of varying patterns of remote memory loss following MTL damage in humans and other animals. Standard consolidation theory (SCT) states that the role of the MTL in memory, particularly that of the hippocampus, is time-limited; the MTL is needed only until consolidation of the memory trace in the neocortex is complete (Milner, Squire, \& Kandel, 1998; Squire, Cohen, \& Nadel, 1984; Scoville \& Milner, 1957; Ribot, 1881). By this view, episodic and semantic memory are believed to undergo the same process of consolidation so that eventually both can be retained and retrieved without hippocampal involvement (Bayley, Hopkins, \& Squire, 2006; Squire, Stark, \& Clark, 2004). Temporally graded memory loss observed in humans (Reed \& Squire, 1998; Zola-Morgan, Squire, \& Amaral, 1986) and other animals with MTL lesions (Squire, 1992) lent support to this view.

Re-examination of the evidence, however, has revealed that the data were not as supportive of SCT as they may have appeared initially. In particular, in humans with MTL damage, loss of autobiographical episodic memory, but not semantic memory, often extends for decades, well 
beyond the time that it is biologically plausible for consolidation to occur (Nadel \& Moscovitch, 1997, 2001; Fujii, Moscovitch, \& Nadel, 2000; Sanders \& Warrington, 1971). Multiple trace theory (MTT) was proposed to account for this evidence. According to MTT, detailed memories of autobiographical events continue to depend on an ensemble of hippocampal-neocortical neurons that represent the memory trace of the event for as long as it exists (Nadel \& Moscovitch, 1997). By contrast, semantic memories are created independently or emerge from the gradual abstraction in the neocortex of commonalities across episodes. They may benefit from the hippocampus and related MTL structures initially, before they are integrated fully with existing information in the neocortex. Consequently, large lesions of the hippocampus and related MTL structures lead to episodic memory loss that can extend for a lifetime, with smaller lesions leading to less extensive loss. With respect to semantic memory, the effects of MTL lesions are more variable and time-limited.

Many recent patient studies support this alternative view. They show that memory loss is more severe for autobiographical episodic memory than for semantic memory, with severe and extensive retrograde amnesia for autobiographical episodes resulting from damage restricted to the MTL (Maguire, Nannery, \& Spiers, 2006; Steinvorth, Levine, \& Corkin, 2005; Cipolotti et al., 2001; Fujii et al., 2000; Moscovitch, Yaschyshyn, Ziegler, \& Nadel, 2000; Viskontas, McAndrews, \& Moscovitch, 2000). Volumetric analysis further suggests that the severity of autobiographical, but not semantic, memory loss is related to hippocampal volume (Gilboa et al., 2005) or metabolism (Eustache et al., 2004) in people with Alzheimer's disease (but see Bright et al., 2006; Kopelman et al., 2003). Finally, functional neuroimaging studies show hippocampal activation that is equivalent for retrieval of recent and remote autobiographical episodes (Viard et al., 2007; Addis, Moscovitch, Crawley, \& McAndrews, 2004; Gilboa et al., 2004; Maguire, 2001; Ryan et al., 2001).

A major dispute is whether damage to the MTL alone, and hippocampus in particular, can lead to episodic memory loss extending to the most remote time periods, as claimed by MTT (cf. Squire \& Bayley, 2007; Moscovitch et al., 2005, 2006). At issue is the precision of neuroanatomical and sensitivity of behavioral measures used to characterize the patients. Those focusing on neuroanatomical evidence have argued, contrary to MTT, that damage restricted to the hippocampus leads only to temporally graded loss; it extends to the most remote time periods only when the lesions encroach on neocortical structures outside of the MTL (Bright et al., 2006; Bayley, Gold, Hopkins, \& Squire, 2005; Gold \& Squire, 2005; Squire et al., 2004; Kopelman et al., 2003). The opposing view, however, is that the variance in methods used to measure AM may account for the differences across studies and not just the extent of extrahippocampal neocortical involvement. Measures of remote memory that credit the number and type of details, rather than ratings of the richness of the memory, may prove to be more sensitive indicators of the extent of autobiographical episodic memory loss (Steinvorth et al., 2005; Levine et al., 2002).

To help resolve this dispute, what is needed is sensitive examination of event details and qualitative ratings of episodic recall, together with whole-brain volumetric analysis and detailed examination of MTL and extra-MTL neocortical structures in amnesic patients. Here we present a detailed investigation of retrograde amnesia in a group of patients with varying amounts of damage to the hippocampus and related MTL structures to evaluate the importance of these regions to AM. To address the behavioral component, we applied a more objective, parametric, text-based scoring procedure that uses the same criteria for scoring remote memories as is used for scoring standardized, narrative-based tests of anterograde memories, where the number of details per event is counted (e.g., Logical Memory subtest of the Wechsler Memory Scale; Wechsler, 1987). In addition, the details are categorized according to whether they are unique to the episode, which distinguishes episodic from generic or semantic content of the memory (Levine et al., 2002). To add another measure of objectivity, scoring was blind, a feature that rarely exists in studies of amnesic memory. At the neuroanatomical level, whole-brain volumetric analysis and detailed examination of MTL and extra-MTL neocortical structures helped to determine the relative contributions of brain damage within and beyond the MTL to remote AM deficits in the patients. If episodic recollection of unique personal events depends on the extent of damage to the system as MTT would predict, we should find autobiographical episodic memory, but not personal semantic memory, to be affected most in patients with complete damage to the MTL bilaterally, independent of the extent of damage to extra-MTL regions. On the other hand, the SCT of hippocampal function would be favored if retrograde memory is found to be temporally graded for both episodic and semantic details, regardless of the extent of MTL damage but related to neocortical damage. In addition, within the MTL, we attempt to determine the relative contributions of hippocampal and extra-hippocampal lesions to patterns of AM loss.

\section{METHODS \\ Participants}

\section{Amnesic Patients}

Four amnesic patients with confirmed MTL lesions participated in this study (Table 1, demographic characteristics; Table 2, neuropsychological data). Patient S. J. had damage, believed to be related to a bacterial infection, that was extensive in the hippocampus bilaterally, but was minimal in the neocortex outside of the MTL. 
Table 1. Demographic and Clinical Characteristics of Amnesic Patients

\begin{tabular}{llcccl}
\hline Initials & Etiology & Age (years) & Education (years) & Lesion Onset & Amnesia $^{a}$ \\
\hline S. J. & Bacterial & 50 & 18 & 1999 & Extensive anterograde; extensive, ungraded retrograde \\
R. G. & Encephalitis & 47 & 14 & 1999 & Extensive anterograde; moderate retrograde \\
C. B. & Encephalitis & 45 & 18 & 2001 & Minimal anterograde; extensive, ungraded retrograde \\
D. A. & Encephalitis & 50 & 17 & 1993 & Extensive anterograde; graded retrograde \\
\hline
\end{tabular}

${ }^{\mathrm{a}}$ Based on clinical observations.

The other three patients had extensive brain damage, secondary to herpes simplex encephalitis, that included the MTL, with the left side affected most in one of the patients (R. G.) and the right side affected most in the remaining two patients (C. B. and D. A.).

S. J., a male physician with 18 years of education, was 50 years old at the time of testing. His case was detailed in a recent article by Gagnon, Foster, Turcotte, and Jongenelis (2004) and is described only briefly here. S. J.'s amnesia was believed to result from a Staphylococcus bacterial infection introduced through a lumbar steroid injection in 1999 for the relief of lower back pain. He was admitted to the hospital, where he experienced an acute hyperglycemic event and tonic-clonic seizures. Neuropsychological testing indicated relatively isolated memory impairment in the context of preserved function in other cognitive domains (Table 2).

R. G. was a 47-year-old man with 14 years of education who was recently included in a group study investigating repetition priming in amnesia (Schnyer, Dobbins, Nicholls, Schacter, \& Verfaellie, 2006). R. G. was admitted to the hospital in November 1999 for treatment of herpes encephalitis. Computed tomography (CT) and magnetic resonance imaging (MRI) revealed a 2 -cm left MTL lesion, with some edema and mass effect. Neuropsychological evaluation indicated severe anterograde and retrograde amnesia and executive dysfunction. Initial discharge at 1 month was unsuccessful due to R. G.'s lack of familiarity with his home and wife of 2 years. Follow-up CT revealed bilateral temporal involvement, with low attenuation in the medial aspects of the left temporal lobe, but a decrease in size of lesion relative to the earlier exam and only minimal right MTL involvement. This was accompanied by modest improvements in procedural learning and retrograde memory but continued anterograde memory impairment. He was discharged to a residential rehabilitation program.

C. B. was a 45-year-old woman with 18 years of education, including a Master's of Social Work. In June 2001, she was treated for herpes encephalitis after MRI showed damage to the right temporal lobe. She was discharged home soon after. In the early stages of recovery, she had difficulty recalling the locations and visual identity of several previously well-known places but benefited from reminders. She also reported that she is missing autobiographical information, and when reminded of past episodes, she experiences only vague recollection of the event.

D. A. was a 47-year-old man with 17 years of education who became amnesic after contracting herpes encephalitis in July 1993. A CT scan taken soon after his diagnosis revealed bilateral MTL hypodensity that was more pronounced on the right. A second scan acquired in the postacute phase showed hypodensity that occupied most of the right anterior temporal lobe, including the medial aspect, but that was minimal on the left. This was accompanied by anterograde and retrograde memory loss.

\section{Control Participants}

Comparisons for the neuroimaging and behavioral portions of the study were made with two separate groups of control participants (Group 1: $n=8,3$ men; Group 2: $n=12,6$ men). All were right-handed, native English speakers, without a history of neurological or psychiatric illness, free from medication known to affect cognitive functioning, and matched in terms of age (Group 1: $M=$ 53.75, $S D=7$; Group 2: $M=52.7, S D=4.07)$ and education (Group 1: $M=16.25, S D=4.62$; Group 2: $M=$ $15.6, S D=1.97)$. A subset of the first control group was included in a separate detailed analysis of MTL structures $(n=4,3$ men, mean age $=52.8$ years, $S D=8.1)$.

Results were analyzed with a modified $t$ test method that treats an individual patient as a sample, thereby permitting comparison of the patient's test score against norms derived from control samples of small to moderate size (Crawford \& Howell, 1998). The patients and controls gave informed, written consent to be involved in the study as approved by the Human Research Ethics Committee of the University of Western Australia for S. J.; the Boston University Medical Center, the Boston VA Healthcare System and Massachusetts General Hospital for R. G. and C. B.; and the Baycrest and the University of Toronto Ethics Committees for D. A.

\section{Experimental Procedure}

\section{MRI Acquisition}

R. G. and C. B. were imaged at the MGH-NMR Center in Charlestown, MA using a 1.5-T MR system (Siemens Sonata) with a gradient coil able to produce pulses of 
Table 2. Selected Neuropsychological Test Scores for the Amnesic Patients

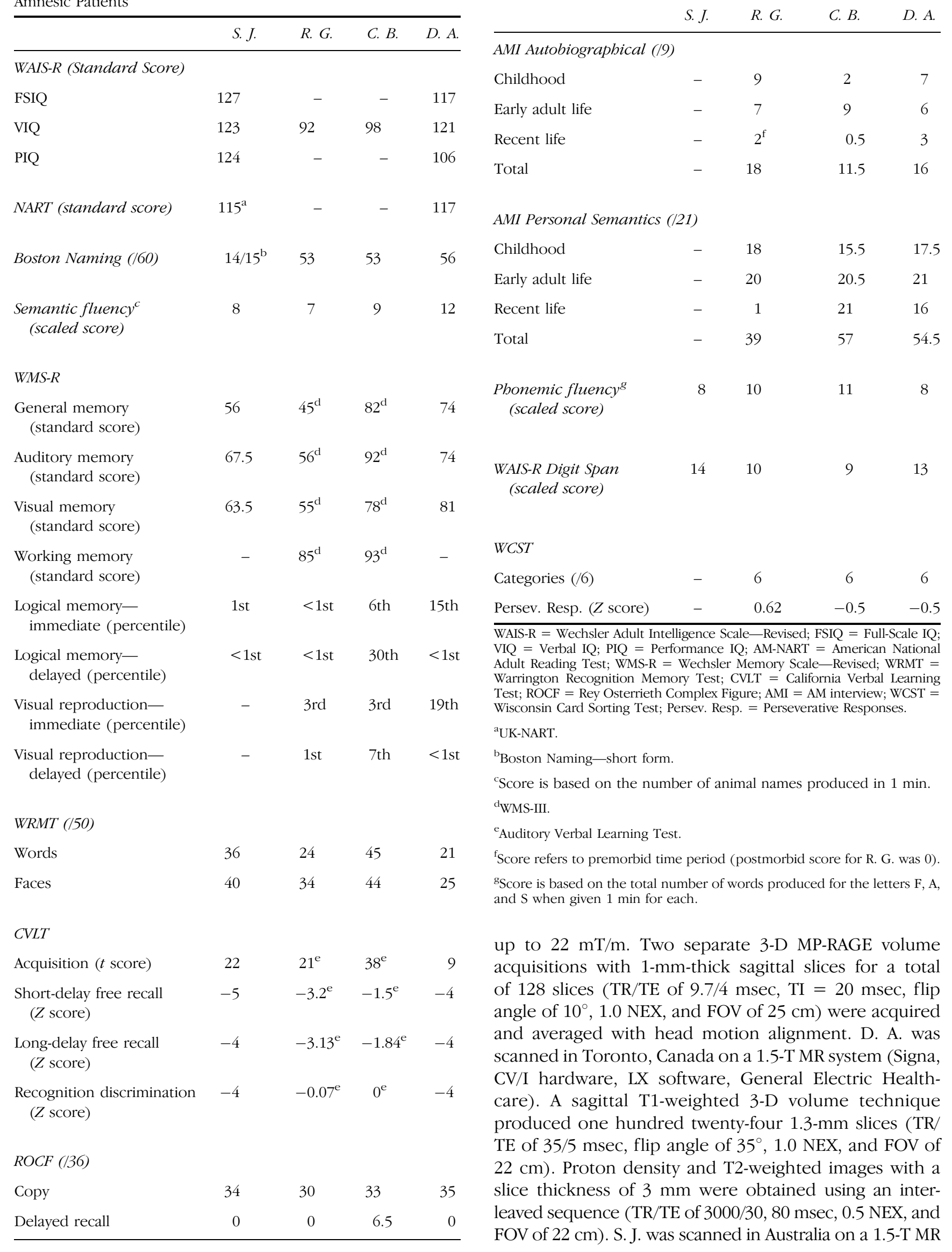

Table 2. (continued) ( 16 
system (Siemens Vision). A T1-weighted technique produced 5-mm-thick sagittal slices (TR/TE of 665/14 msec, flip angle of $70^{\circ}, 2.0 \mathrm{NEX}$, and FOV of $23 \mathrm{~cm}$ ). Axial proton density and T2-weighted images with a slice thickness of $5 \mathrm{~mm}$ were obtained using an interleaved sequence (TR/TE of 3000/17, $102 \mathrm{msec}, 2.0 \mathrm{NEX}$, and FOV of $28 \mathrm{~cm}$ ). Coronal and temporal lobe FLAIR images were acquired with slice thicknesses of $5 \mathrm{~mm}$ and $3 \mathrm{~mm}$, respectively (TR/TE 9999/110, TI 2360 and $2400 \mathrm{msec}$, $1.0 \mathrm{NEX}$, and FOV of $24 \mathrm{~cm}$ ). Diffusion-weighted imaging was acquired with a slice thickness of $5 \mathrm{~mm}$ (TR/TE 118/ $23.5 \mathrm{msec}, 1.0 \mathrm{NEX}$, and FOV of $23 \mathrm{~cm}$ ).

\section{Whole-brain Analysis}

MRI data were analyzed via an updated version of our image processing pipeline (Dade et al., 2004; Kovacevic et al., 2002). The main modification to this protocol involved template matching, allowing for comparison of individual images to a standard image and facilitating automation of previously semiautomated steps. The first step in the pipeline was to create an unbiased nonlinear average of T1-weighted images from the nine matched comparison participants using a modification of an algorithm previously developed for mouse brain MRI (Kovacevic et al., 2005). Each participant's T1-weighted image was then registered to the template brain (Woods, Grafton, Holmes, Cherry, \& Mazziotta, 1998; Woods, Grafton, Watson, Sicotte, \& Mazziotta, 1998), preserving the original size of the brain while standardizing the position and orientation. Images were resampled into template space using windowed sinc interpolation. Template matching was accomplished via nonlinear registration of T1-weighted images to the template image (Collins \& Evans, 1997).

Removal of nonbrain tissue from the image incorporated thresholding information derived from the proton density (PD)- and T2-weighted images, facilitating the distinction between dura mater and gray matter (Kovacevic et al., 2002; this refinement was not performed for C. B. and R. G., who did not have PD- and T2-weighted images). This is contrasted to methods of brain extraction on the basis of the T1-weighted image that emphasize the cortical surface, inconsistently preserving subdural cerebrospinal fluid. Focal lesions appearing as T1 hypointensities in D. A., R. B., and C. G. were visualized and defined using Analyze software (Biomedical Imaging Resource, Mayo Foundation, Rochester, MN, USA). The area of damage was determined by detailed slice-by-slice visual inspection on axial views by a radiologist (F. G.). In order for a lesion to be traced, it had to appear on more than one slice, with a diameter of at least $3 \mathrm{~mm}$ on one of the slices. The boundary of the lesion was manually delineated on each MR T1-weighted axial slice using the Analyze region-of-interest (ROI) module. A 3-D lesion ROI for each patient was produced by combining all lesion tracings from each slice.
The voxels on the T1-image were then classified as representing gray matter, white matter, or cerebrospinal fluid using a robust automated tissue classification method that corrects for radio-frequency inhomogeneity inherent to MR scanning (Kovacevic et al., 2002). We report gray matter volumes. As our segmentation protocol is flexible across different T1-weighted contrasts, no adjustment was necessary to accommodate images acquired from different scanners. Voxels within lesioned areas were classified as lesion tissue. S. J.'s lesions, appearing as T2 hyperintensities, were not reclassified for the wholebrain analysis but were masked out for our detailed analyses of MTL substructures (see below). A modified Semi-Automated Brain Region Extraction (SABRE; Dade et al., 2004) method was then used to create ROIs on the template brain. Based on identification of the edges of the brain and the anterior and posterior commissures, a Talairach-like (Talairach \& Tournoux, 1988) grid is automatically created. The algorithm uses this grid along with the 15 manually identified landmark coordinates and tracing of the cingulate gyrus to divide the brain into 38 regions (19 per hemisphere).

Nonlinear deformation field matching of the template to individual images was used to customize these regions to fit each participant's brain anatomy (rather than transforming images to fit the template, which can distort interindividual topographical variability). For reasons of simplicity and the small $n$, data were collapsed across a subset of the 38 regions to yield 20 regions (10 per hemisphere) as follows: ventral prefrontal, dorsal prefrontal, anterior temporal, medial temporal, posterior temporal, anterior cingulate, posterior cingulate/retrosplenial, inferior parietal, superior parietal, and occipital (see Figure 1). All images were manually inspected, slice-by-slice, to confirm the accuracy of pipeline steps. Regional gray matter volumes were adjusted for total intracranial capacity using a regression-based method (Arndt, Cohen, Alliger, Swayze, \& Andreasen, 1991). Our tissue compartment segmentation and SABRE software are particularly well suited to analysis of nonnormal brains, as they do not require spatial transformation that can distort interindividual topographical variability. These algorithms have been applied to normal aging (Dade et al., 2004), multiple sclerosis (Feinstein et al., 2004), and dementia (Gilboa et al., 2005).

\section{MTL and Posterior Cingulate/Retrosplenial Cortex Analysis}

The MTL region in our automated SABRE protocol contains entorhinal, perirhinal, and parahippocampal cortices, in addition to the hippocampus. A more detailed examination of these structures was accomplished using manual tracing on the normalized and coregistered T1-weighted images according to Insausti et al. (1998) for the hippocampus, entorhinal cortex, and perirhinal cortex and according to Callen, Black, Gao, 
Figure 1. Regional divisions as defined by a modification of the SABRE software (Dade et al., 2004). VF = ventral frontal; DF = dorsal frontal; $\mathrm{AT}=$ anterior temporal; $\mathrm{PT}=$ posterior temporal; $\mathrm{MT}=$ medial temporal; $\mathrm{IP}=$ inferior parietal; $\mathrm{SP}=$ superior parietal; $\mathrm{PCG}=$ posterior cingulate/ retrosplenial; ACG $=$ anterior cingulate.

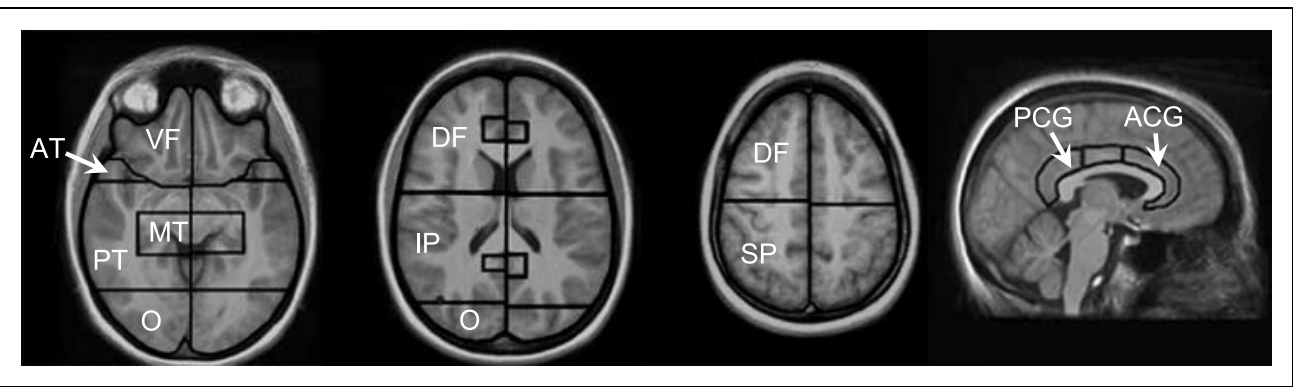

Caldwell, and Szalai (2001) for the parahippocampal cortex and, in supplementary analyses, for the posterior cingulate/retrosplenial cortex. These gray matter volumes were compared to those of four control subjects matched to the patients for age, education, and sex. Patient S. J.'s hippocampal ROIs contained nonfunctional tissue, as indicated by hyperintensities restricted to these same ROIs on the T2-weighted FLAIR images. His hippocampal tissue volumes were therefore corrected by masking out nonfunctional tissue as measured on the T2-weighted FLAIR images.

\section{Autobiographical Interview}

The autobiographical interview (Levine et al., 2002) was administered for a fine-grained analysis of the patients' ability to retrieve, under different levels of retrieval support, autobiographical memories established at different times in their lives. Participants were asked to provide details of a significant one-time episode that was personally experienced at a specific time and place from each of five life periods (childhood, to age 11; adolescence, ages 11-17; early adult life, ages 18-30; middle adult life, ages 30-45; and the past year). In cases where a specific event was not generated independently, an extensive list of event topics was presented to assist in retrieval.

In order to examine facilitative effects of retrieval support on memory, we manipulated the level of structure available to participants across three conditions: recall, general probe, and specific probe (see Levine et al., 2002). At recall, participants spoke about the event extemporaneously without any interruption from the examiner, continuing until it was evident that they had reached a natural ending point. After an event was recalled, general probes were used to clarify instructions and to encourage greater recall of details. If general probing did not elicit a specific event, the participant was given the option of selecting a different event that was more likely to result in successful recall. General probes were limited to nonspecific statements or repetitions of the instructions. At the specific probe phase, a structured interview was administered that was designed to elicit additional contextual details. In order to prevent the specific probe process from contaminating recall of subsequent memories, specific probing was administered after all five events were recounted under the recall and general probe conditions.

Descriptions were recorded, transcribed, and verified, when possible, by relatives or friends of the participants. Following administration, event descriptions were segmented into details, which are informational bits relating to a one-time occurrence, observation, or thought that are often expressed as a grammatical clause. Details were classified as "internal" or episodic and assigned to one of five categories (event, place, time, perceptual, and emotion/thought) if they related directly to the main event described, were specific to time and place, and conveyed a sense of episodic re-experiencing. Otherwise, details were considered "external," consisting of autobiographical events tangential or unrelated to the main event, semantic facts, repetitions, or other metacognitive statements or editorializing. The sum of details in each category was calculated in a cumulative manner for each level of cueing.

Quantitative ratings were accompanied by qualitative ratings assigned to each of the internal detail categories, with the possibility of attaining a maximum of 3 points for each category (event, time place, perceptual, emotion/ thought). Three points were assigned when the description was rich, highly specific, and appeared to emerge from a feeling of re-experiencing. Two points were assigned to detailed descriptions falling short of a 3-point description. One point was assigned to descriptions containing general, nonspecific information but still episodic in nature. No points were assigned when there was no information pertaining to the specified category, or for responses on the basis of semantic knowledge rather than episodic memory. Episodic richness (the overall degree to which a feeling of re-experiencing was conveyed) was rated on a similar scale that was extended to 6 points to provide a finer-grained rating and to account for the greater importance of this category relative to the others. The time integration rating (on a scale of $0-3$ ) was meant to gauge a person's ability to integrate the recalled episodic event into a larger time scale by giving additional temporal contextual information or relating it to other life periods. 
Although ratings in the first four categories were mutually exclusive (i.e., aspects of a memory could not be counted in more than one category), the episodic richness and time integration categories were based on an overall assessment of the event. The sum of the ratings pertaining to episodic re-experiencing formed the ratings composite $(\max =21)$. Three extensively trained scorers who had achieved high interrater reliability (see Levine et al., 2002) and who were blind to the group were assigned memories at random for scoring. Interrater reliability was further addressed by scoring patients' memories by two separate raters; discrepancies (which were minor) were resolved by discussion.

\section{RESULTS}

\section{Neuroimaging}

\section{Whole-brain Analysis}

MR images for S. J. are presented in Figure 2. Each of the other three patients is presented in Figure 3. Figure 4 shows the volumes of the major neocortical divisions on the basis of the SABRE analysis. In all four cases, volume loss was greatest in the MTL. However, additional volume loss was noted in other regions, most often in the anterior temporal and anterior cingulate regions.

S. J. showed significant bilateral MTL volume loss. It should be noted, however, that the whole-brain analysis yielded an overestimate of functional tissue in S. J.'s MTL relative to the more detailed analysis, where it was possible to mask out nonfunctional tissue (see Methods and below). Additional minor volume loss was noted over the left inferior parietal cortex, the left posterior cingulate/retrosplenial cortex, the right anterior and posterior temporal cortex, and the right anterior cingulate cortex. R. G. had a large left temporal lesion, causing marked volume loss over all left temporal lobe sectors, with encroachment onto the left ventral frontal cortex. Left anterior and posterior temporal volumes were also reduced. R. G.'s right temporal lobe was also affected, especially in the medial and posterior regions as well as a minor loss in the anterior region. R. G. showed elevated superior parietal and dorsal frontal volumes owing to a combination of his head shape (larger in the superior-inferior plane) and the automated placement of the line dividing the superior from the inferior parietal regions. Accordingly, R. G. had relatively lower inferior parietal volumes. C. B. had focal damage to the right temporal lobe, affecting anterior, medial, and posterior regions. The right inferior parietal cortex was only slightly affected. Left temporal volume loss was restricted to the medial region. D. A. showed a rightlateralized pattern of volume loss, maximal over the anterior temporal and MTL regions, which were ablated by large focal lesions. Volume loss was also observed over other right-hemispheric regions, especially the posterior temporal, ventral frontal, and occipital regions, and also the anterior cingulate. Left-lateralized volume loss was restricted to the MTL region. Small lesions to right posterior thalamus and the left middle temporal gyrus of approximately $3 \mathrm{~mm}$ and $10 \mathrm{~mm}$ in diameter, respectively, were also observed.

\section{MTL and Posterior Cingulate/Retrosplenial Cortex Analysis}

Figure 5 shows MTL volumes based on detailed ROI analysis. S. J. had profound volume loss in the hippocampus bilaterally. This was accompanied by mild volume loss in the left entorhinal cortex and the right parahippocampal cortex, and more significant volume loss in the left parahippocampal cortex. R. G. had hippocampal damage that was equally severe to that of S. J.'s on the left but less severe on the right. However, R. G. showed the greatest loss of volume in bilateral perirhinal, entorhinal, and parahippocampal cortices. C. B.'s hippocampus was reduced to a greater extent on the right than on the left. A similar pattern of loss was

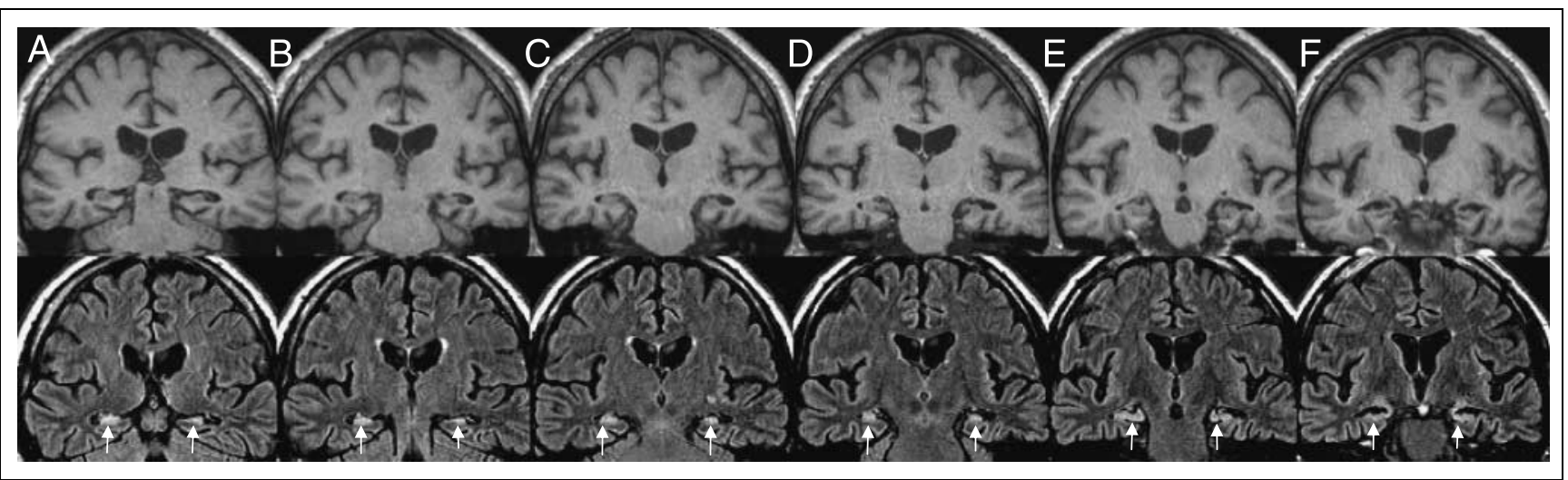

Figure 2. Coronal T1-weighted (top row) and FLAIR (bottom row) MR images corresponding approximately to each T1 MRI from posterior (A) to anterior (F) showing bilateral hippocampal lesions in S. J. Images are presented according to radiological convention (right side of brain on left side of image). Reduced volume in hippocampi bilaterally on T1 images indicates atrophy. Hyperintensities spreading along the entire hippocampus (arrows) on FLAIR images indicate extensive bilateral hippocampal lesions. 


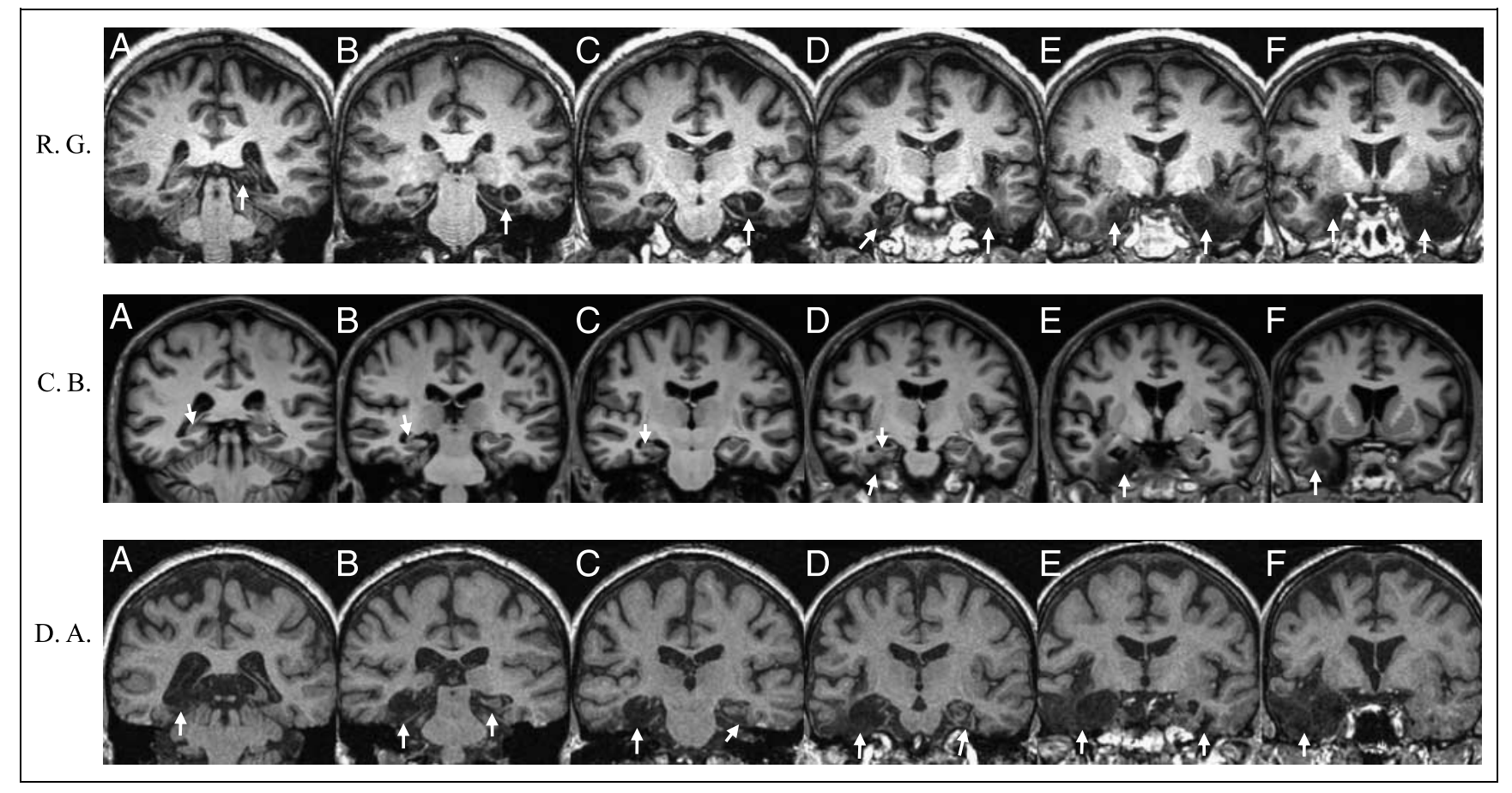

Figure 3. Coronal T1-weighted MR images from posterior (A) to anterior (F) showing the locus and extent of MTL damage in R. G. (top row), C. B. (middle row), and D. A. (bottom row). Hypointense signal indicates damaged tissue. Images are presented according to radiological convention (right side of brain on left side of image). See text for detailed description of the lesions. R. G. has bilateral MTL damage, mainly on the left. Left perirhinal, entorhinal, and parahippocampal cortices, as well as the anterior temporal lobe, are mostly destroyed (right arrows). The left hippocampus is damaged by over $90 \%$. Right perirhinal and entorhinal cortices are also severely damaged (left arrows in images D-F). C. B.'s MTL damage is mostly on the right. Images A-D show that the right hippocampus (downward arrows) is much smaller than the left, and the right perirhinal, entorhinal, and parahippocampal cortices are damaged (upward arrows in images D and E). The right anterior temporal lobe is also damaged (arrow in image F). D. A. has bilateral MTL damage, mainly on the right. Images A-F show that the right perirhinal, entorhinal, and parahippocampal cortices, as well as the anterior temporal lobe, are mostly destroyed (left arrows). The right hippocampus is damaged by over $90 \%$. Left perirhinal, entorhinal, and parahippocampal cortices are also severely damaged (right arrows in images B-E).

apparent in perirhinal and entorhinal cortices, whereas the parahippocampal cortex was only marginally outside the range of controls on the right and intact on the left. Finally, volume loss in D. A.'s hippocampus was equal in extent to that observed in S. J. on the right and was close to it on the left, though residual tissue remains. D. A. also had severe reductions bilaterally in the perirhinal, entorhinal, and parahippocampal cortices.

According to the SABRE analysis, S. J. had marginally reduced left posterior cingulate/retrosplenial volume. On the right, his volume in this region was at the edge of the comparison group's confidence interval, as was the case for $\mathrm{C}$. B. on the left. As these two regions are specifically implicated in AM (Svoboda et al., 2006; Maguire, 2001; Valenstein et al., 1987), we conducted ancillary manual tracing analyses of these regions using methods as specified by Callen et al. (2001). Patients were compared to the same four matched comparison subjects as used in the other manual tracing analyses. By this analysis, Patients C. B. and D. A. showed mild rightlateralized volume loss, at the edge of the comparison subjects' distribution, whereas S. J. showed mild leftlateralized volume loss, again at the edge of the comparison subjects' distribution.

\section{Autobiographical Interview}

The total number of details collapsed across the life periods was analyzed separately for the internal and external detail categories and for the ratings composite scores to give an overall impression of impairment in the patients. Because there were no appreciable differences between the free recall and general probing conditions, the two were collapsed into a low retrieval support condition (i.e., recall) and analyzed separately from a condition of high retrieval support (i.e., specific probe; Levine et al., 2002). The quantitative and qualitative composite scores for each level of retrieval support were then separated into five life periods to determine any effects of age of memory. The number of internal (episodic) and external (nonepisodic, mostly personal semantic) details are displayed in Figure 6.

\section{Composite Measures of Autobiographical Retrieval}

Examination of the composite scores for each of the internal, external, and ratings categories indicates that the patients differed from controls with respect to internal detail generation depending on the type of analysis. 


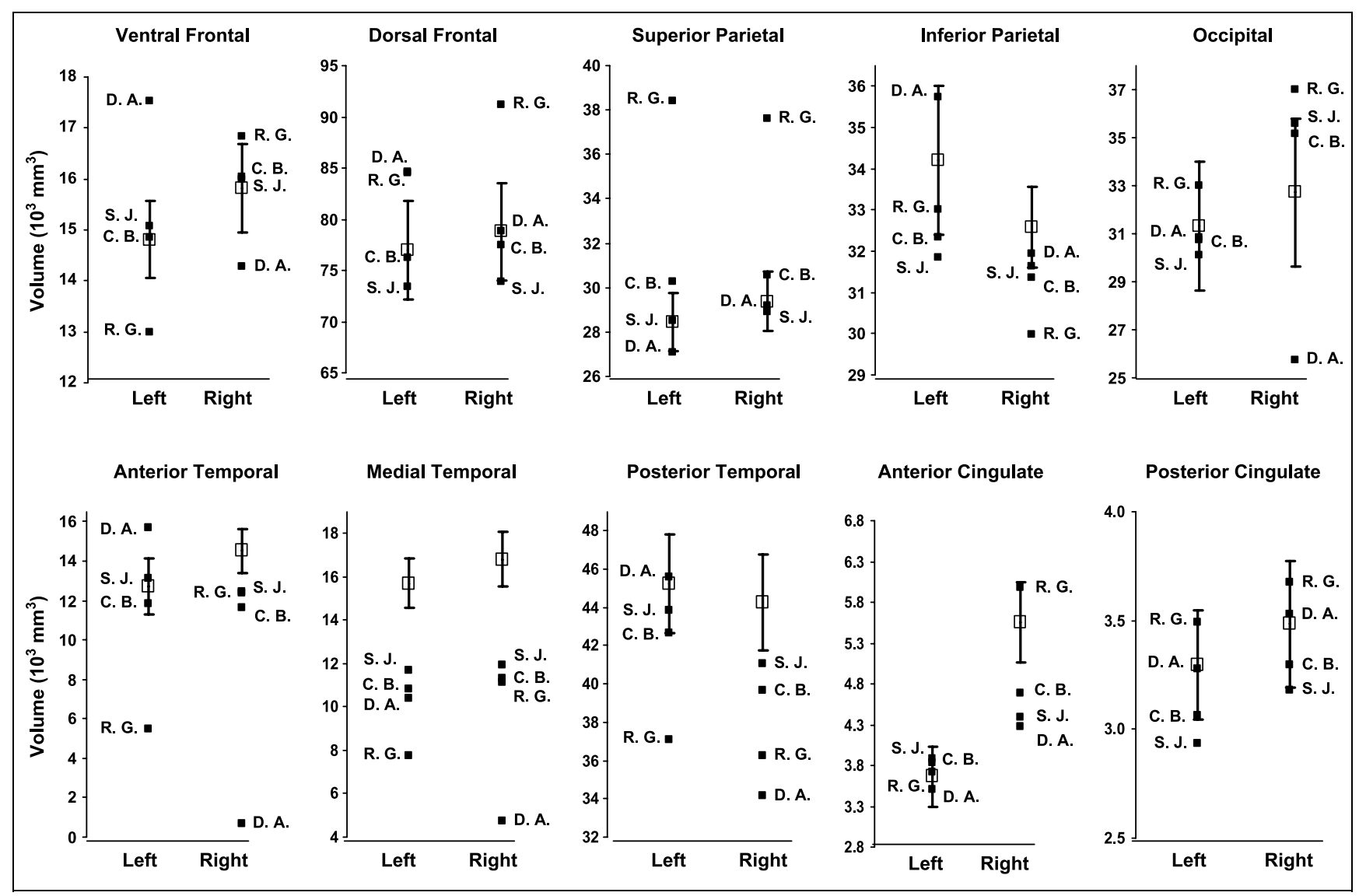

Figure 4. Regional gray matter volumes, as defined by SABRE, plotted for the amnesic patients in comparison to the control group. Patients are represented by filled squares and control group mean is represented by unfilled squares. Error bars indicate $95 \%$ confidence intervals for control group.

Significant differences in performance were observed in S. J. and C. B. for episodic details based on scores from quantitative (internal) and qualitative (ratings) analyses, but not for external details, under recall [S. J.: internal, $t(11)=-2.34, p=.02$, ratings, $t(11)=-4.02, p=.001$; C. B.: internal, $t(11)=-1.8, p=.05$, ratings, $t(11)=$ $-4.46, p<.001$ ] and specific probing [S. J.: internal, $t(11)=-2.34, p=.02$, ratings, $t(11)=-4.02, p=.001$; C. B.: internal, $t(11)=-1.8, p=.05$, ratings, $t(11)=$ $-4.46, p<.001]$. Scores for D. A. approached significance for internal details and reached significance for ratings when specific probing was used $[t(11)=-1.58, p=.07$; $t(11)=-1.79, p=.05$, respectively], whereas those for $\mathrm{R}$. G. approached significance only for ratings during recall $[t(11)=-1.566, p<.07]$.

\section{Life Period Analysis of Autobiographical Retrieval}

A finer examination of detail composites and ratings within each life period shows that all patients exhibited difficulty producing episodic details relative to controls, but to a varying extent depending on the life period and level of cueing (presented in Figure 6). Under recall, C. B. retrieved significantly fewer internal details for the childhood period $[t(11)=-1.907, p=.04]$, although ratings indicated additional impairment for the other four life periods [childhood: $t(11)=-3.08, p=.005$; adolescence: $t(11)=-1.55, p<.01$; early adulthood: $t(11)=-3.13, p=.005$; mid-adulthood: $t(11)=-2.15$, $p<.03$; recent years: $t(11)=-4.42, p=.001] . \mathrm{R} . \mathrm{G}$. differed significantly from controls when performance was based on ratings for early adulthood $[t(11)=-2.76$, $p=.009]$ and mid-adulthood $[t(11)=-2.15, p<.03]$. D. A. displayed worse performance than controls in recalling a memory from the most recent time period, which took place after he became amnesic, that was of marginal significance when based on internal details $[t(11)=-1.64, p=.065]$ and significant when based on ratings $[t(11)=-2.67, p=.01]$. S. J. showed the greatest impairment overall, producing significantly fewer internal details for childhood $[t(11)=-2.97, p=$ $.005]$, early adulthood $[t(11)=-2.12, p<.03]$, and mid-adulthood memories $[t(11)=-1.82, p<.05]$; this pattern of significance was reproduced in the ratings $[t(11)=-4.49, p<.001 ;-4.58, p<.001 ;-4.6, p<$ .001, respectively]. His deficit for internal details for the most recent time period approached significance $[t(11)=-1.64, p=.07]$. All patients performed within the range of healthy controls in retrieving external details for all life periods. 


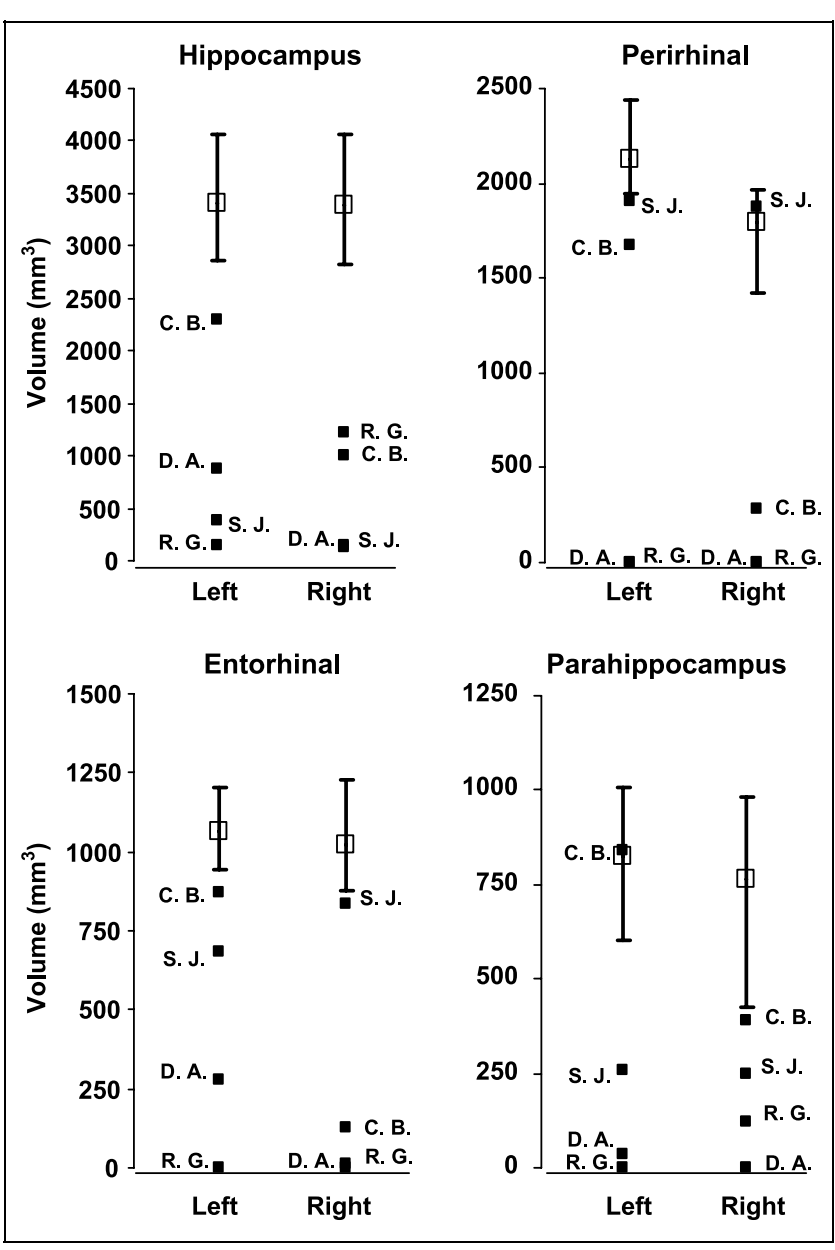

Figure 5. Gray matter volumes of MTL regions plotted for the amnesic patients in comparison to four controls. Patients are represented by filled squares and control group mean is represented by unfilled squares. Error bars indicate range of control subjects' volumes.

A more striking pattern of impairment was observed in S. J., R. G., and D. A. when the number of details was summed across the three retrieval conditions (i.e., specific probing). S. J. had difficulty providing an autobiographical episodic memory specific to time and place for any time period sampled in response to specific probing. The results were significant for internal detail generation and ratings for childhood [internal: $t(11)=-3.12, p=$ .005 ; ratings: $t(11)=-6.04, p<.001]$, early adulthood [internal: $t(11)=-1.8, p<.05$; ratings: $t(11)=-2.96$, $p=.007$ ], mid-adulthood [internal: $t(11)=-1.82, p<$ .05 ; ratings: $t(11)=-6.37, p<.001$ ] , and recent (postmorbid) time periods [internal: $t(11)=-1.77, p=.05$; ratings: $t(11)=-3.22, p=.004]$, and there was a trend toward significance for internal details retrieved in relation to a memory from adolescence $[t(11)=-1.7, p=$ .059]. Results indicated significantly impoverished recollection for R. G. for the mid-adulthood period based on scores for internal details $[t(11)=-2.03, p=.03]$ and ratings $[t(11)=-3.89, p=.001]$ when given specific probes, whereas D. A. showed a specific deficit for early adulthood and recent (postmorbid) time periods for internal details [early adult: $t(11)=-1.89, p=.04$; recent: $t(11)=-2.22, p=.02$ ] and ratings [early adult: $t(11)=-4.82, p<.001$; recent: $t(11)=-6.19, p<$ .001]. By contrast, C. B.'s autobiographical episodic memory improved to control levels with cueing for most time periods. There was evidence of impoverished recollection relative to controls that approached significance for internal details of the most remote and most recent (postmorbid) time periods $[t(11)=-1.65, p=$ $.06 ; t(11)=-1.72, p<.06$, respectively] and that was significant for ratings of early and mid-adulthood memories $[t(11)=-2.02, p=.03$ and $t(11)=-2.64, p=.01$, respectively]. As in the recall condition, nonepisodic detail generation remained similar to control levels for all patients. Overall, S. J., who had the greatest volume loss in his hippocampus bilaterally, displayed the most severe autobiographical episodic memory impairment for all time periods, even though volume loss in each of the other MTL and neocortical structures matched or was less than that found in the other patients. The results suggest that the hippocampus contributes uniquely to episodic memory independent of other structures and that it is not simply sheer loss of MTL tissue that accounts for severity of deficit.

\section{DISCUSSION}

The present study examined memory for personal events from recent and remote life periods under varying levels of verbal prompting in four amnesic cases with different patterns of hippocampal and neocortical loss. Quantitative volumetric data confirmed that three of the patients had extensive MTL damage that was unequally distributed between hemispheres, along with damage to neocortical sites that have been implicated previously in AM. Nevertheless, the two patients with the greatest extrahippocampal MTL damage and significant neocortical damage (R. G. and D. A.) presented with the least retrograde memory impairment. Memory loss was restricted to the time period just before the onset of amnesia and, in the case of D. A., to a postmorbid time period; memories from the most remote time periods were spared. The most severe and extensive loss of these memories, however, was observed in a fourth patient (S. J.), who had more extensive damage to his hippocampus bilaterally, but whose extra-hippocampal MTL and neocortical damage was no more extensive than in any of the other patients examined, and in many regions less extensive. By contrast, none of the patients was impaired in the retrieval of personal semantic information from the same life events. Together, these findings suggest that the autobiographical episodic memory loss presented by the patients is more likely a function of hippocampal integrity than extra-hippocampal MTL or neocortical involvement. Such findings are inconsistent with the view that the hippocampus plays a 


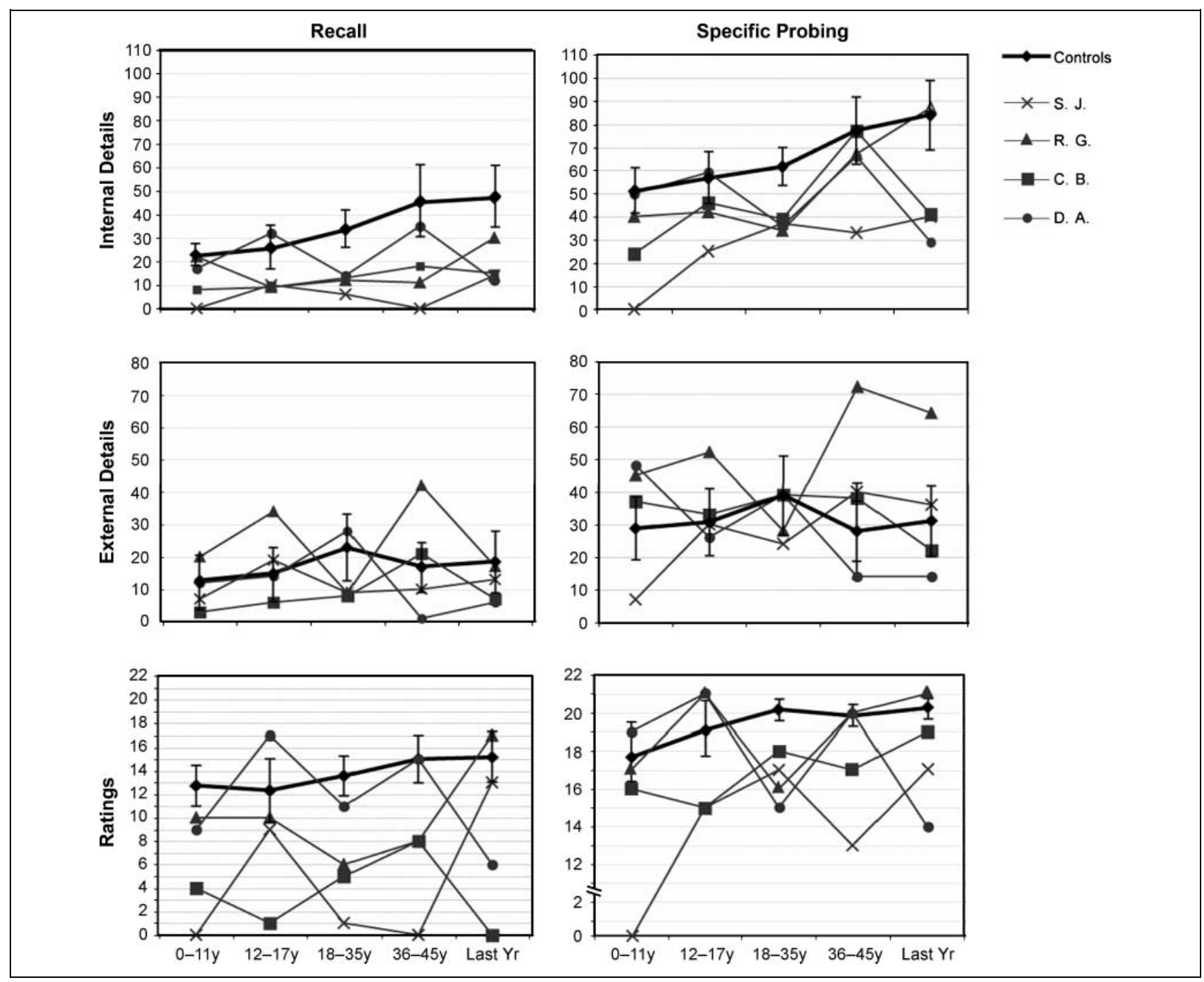

Figure 6. Total number of details retrieved by patients and controls for internal (top row), ratings (middle row), and external (bottom row) categories across five life periods for recall (left column) and after specific probing (right column). All time periods correspond to memories formed premorbidly, with the exception of the two most recent time periods ("36-45 years" and "last year") for D. A. and most recent time period for the other patients, which correspond to postmorbid memories. Error bars indicate $95 \%$ confidence intervals for control group.

time-limited role in both episodic and semantic memory. In what follows, reference is made to results from the specific probing condition of the autobiographical interview, unless otherwise specified, as this condition most effectively differentiated the two types of memories in patients compared to controls.

\section{Hippocampal and Neocortical Contributions to AM}

SCT and MTT make opposite claims with respect to the brain regions necessary for episodic memory. The autobiographical interview allows for direct comparison of these opposing views on episodic memory in people with severe amnesia relating to hippocampal damage with or without accompanying neocortical damage as indicated by detailed volumetric analyses. In the autobiographical interview, episodic memory is indexed by complementary quantitative analysis of internal details and qualitative analysis in the form of ratings to allow for a complete picture of episodic memory integrity. When both internal details and ratings are taken into account, S. J.'s recollection of past personal episodes experienced across his lifetime was severely compromised. The other patients were not as impaired, although they performed poorly relative to controls in retrieving episodes from premorbid time periods, particularly those closer to the onset of their amnesia. All patients had in common impaired retrieval of episodic details relating to the early adulthood time period, but this may have resulted from the low variability among controls for this time period as much as difficulties in accessing these memories even in patients with mild hippocampal damage. C. B.'s recollection of a childhood memory was in the borderline range, whereas R. G. and D. A. showed complete sparing of this memory and one from their teenage years. 
When considered in the context of the patients' lesion profiles, it appears that the degree of impaired remote autobiographical episodic memory in the patients is related to the amount of hippocampal damage observed. $\mathrm{S}$. J. had the most severe impairment and most extensive damage to his hippocampus bilaterally and some left entorhinal and bilateral parahippocampal atrophy, whereas R. G.'s remote memory loss was the mildest and was accompanied by partial hippocampal damage but almost complete loss to extra-hippocampal MTL cortices, including more severe damage to the entorhinal and parahippocampal cortex bilaterally than that observed in S. J. The finding of better recognition than recall on neuropsychological tests of anterograde memory provides further evidence of MTL damage largely restricted to the hippocampus in S. J. (e.g., Yonelinas et al., 2002).

Although D. A.'s hippocampal volume loss was as extensive as S. J.'s on the right and close to it on the left, it is possible that the remaining tissue on the left was sufficient to sustain D. A.'s remote AM, even in the context of additional compromise to other MTL structures. D. A.'s ability to acquire postmorbid memories lends further support to the suggestion that the remaining hippocampal tissue is viable. R. G. and D. A. also showed preserved retrieval of episodic details of a postmorbid memory, whereas the other patients retrieved few details, with the degree of deficit approaching significance for C. B. and reaching significance for S. J. and for D. A. for a second, more recent postmorbid memory. Postmorbid memory acquisition is often viewed as a marker of hippocampal integrity, and as such, provides further evidence that what remains of R. G.'s and D. A.'s hippocampi is functionally viable to a certain extent and that S. J.'s are severely compromised, perhaps more so than the other patients'. It is unclear why D. A. had difficulty retrieving details of an event from the past year but not from mid-adulthood. One possibility is that the constraint of having a single year from which to select an event for the most recent time period, compared to nearly a decade for the mid-adulthood time period, prevented $\mathrm{D}$. A. from selecting a more memorable event that occurred just before the 1-year cutoff. It should be noted that, in general, the patients were free to choose any memory they wished for each time period. Had the event topics been selected for them, as is the case with other procedures (e.g., Kopelman, Wilson, \& Baddeley, 1990), the patients may have recollected far fewer details, even for the most remote time periods.

Although S. J. had evidence of mild inferior parietal atrophy, his relatively preserved performance in cognitive domains outside of memory as indicated by neuropsychological testing suggests that the consequences of this damage are relatively minor. With respect to regions known to be associated with AM (Cabeza \& St. Jacques, 2007; Svoboda et al., 2006; Maguire, 2001), S. J.'s posterior cingulate/retrosplenial cortex on the left was com- parable to loss displayed by C. B. and D. A. in these regions on the right, both marginally below the range of controls. Moreover, there are no reports of unilateral damage in these regions causing amnesia. In addition, C. B., who had less extensive hippocampal damage, but comparable damage to right anterior and posterior temporal cortices, and greater damage to right perirhinal and entorhinal cortices, was impaired for all time periods in response to general probing, but displayed few residual deficits with specific probing. The benefit from contextual cueing observed in C. B. for all time periods, except the most recent one, suggests that her deficit may relate in part to an inability to engage in the strategic retrieval or effortful processing demands of reconstructing details of one's past (McKinnon, Black, Miller, Moscovitch, \& Levine, 2006; Rosenbaum, McKinnon, Levine, \& Moscovitch, 2004; Kopelman et al., 2003). However, no patient had clear damage to the dorsolateral frontal cortex, which has been implicated in "executive" demands, nor did any patient display deficits on neuropsychological tests of executive function.

R. G. and D. A. had the most severe damage to all extra-hippocampal MTL sectors as well as anterior and posterior temporal cortex, bilaterally in R. G. and on the right in D. A. along with the right occipital cortex (Gilboa et al., 2005; Rosenbaum et al., 2004; Rubin \& Greenberg, 1998). Nevertheless, these patients performed similarly to controls for the most remote time periods and would certainly present as normal based on the analyses conducted by Bayley et al. (2005). We do not mean to imply that damage to the extra-hippocampal MTL and the neocortex does not contribute to AM loss, but the current data suggest that it is the location of the lesion, and not only the extent, that is paramount. Taken together, these results suggest that the extent and severity of AM loss is likely to be related more to the size of hippocampal lesions than to additional MTL/ neocortical damage, arguing in favor of MTT.

Despite competing predictions of hippocampal involvement in remote episodic memory, the two theories make similar predictions with respect to its unessential role in remote semantic memories and its obligatory role in the acquisition and maintenance of recent episodic memories. Findings from the present study support both of these predictions. With respect to semantic memory, external details, which are composed mainly of personal "facts" and knowledge about the world that are not tied to any one event, were retrieved with ease by all patients for all time periods. Interestingly, Patient R. G. recalled significantly more external details than did controls for the two most recent time periods, which represent memories formed just previous to and after the onset of his amnesia. This finding lends validity to the autobiographical interview as an effective technique for dissociating episodic from semantic details to which they are intimately tied when the standard method of administration and scoring is followed. 
The overall pattern of impaired remote episodic memory but spared semantic memory has been observed in earlier investigations of unilateral temporal lobe epilepsy or lobectomy (Viskontas et al., 2000) and Alzheimer's disease (Gilboa et al., 2005) using the autobiographical memory interview (AMI; Kopelman et al., 1990). Functional neuroimaging studies are also consistent with the current findings: Hippocampal activation is equivalent for recent and remote events as long as they retain a vivid, experiential component (Viard et al., 2007; Addis et al., 2004; Gilboa et al., 2004; Maguire, 2001; Ryan et al., 2001). Similarly, in animals, there are a number of reports that retrograde amnesia can be extensive and without a temporal gradient, although the conditions that lead to this severe amnesia as compared to the more circumscribed, temporally graded one are unknown (cf. Murray \& Bussey, 2001; Nadel \& Moscovitch, 1997, 2001; Milner et al., 1998). Recent work in rats suggests that if the memory continues to rely on its initial, detailed context, then extensive retrograde loss is observed, whereas a temporal gradient is noted as the memory is transformed from a contextually dependent one to a more generic memory (Moscovitch et al., 2005; Winocur et al., 2005a, 2005b; Rosenbaum, Winocur, \& Moscovitch, 2001; see also Clark, Broadbent, \& Squire, 2005a, 2005b; Martin, de Hoz, \& Morris, 2005).

\section{Comparisons with Other Cases of MTL Amnesia}

Beginning with Moscovitch et al. (2000), autobiographical events retrieved by hippocampal amnesics have been scored by some investigators in terms of the number and type of details recollected, rather than the older method of assigning a fixed number of points to reflect the richness of the memory. In all cases that used the new procedures as detailed in Levine et al. (2002), remote AM loss extended to early childhood. This was true of people whose lesions affected the hippocampus primarily, but not those with frontal or anterior temporal damage, both of whom benefited from prompting (Levine, 2004). These findings are consistent with those observed in the present study.

Additional studies with K. C. and H. M. showed them to be equally impaired in retrieving both internal and external details from all time periods in K. C. (Rosenbaum et al., 2004), and all but one time period in H. M. (Steinvorth et al., 2005). It is also notable that neither patient improved under conditions of high retrieval support. It is this pattern of impairment of both remote episodic and personal semantic memory that we believe is attributed to extensive combined loss of tissue in the hippocampus and surrounding MTL cortices as well as in the lateral temporal neocortex, which was evident in both K. C. and H. M. Impaired retrieval of episodic details, but not semantic details, that, nevertheless, affects all life periods, as observed in S. J., reflects more specifically bilateral hippocampal damage. Interestingly, the memory that was least disrupted in S. J. was also one from his teenage years, the same time period from which $\mathrm{H}$. M. recounted his sole personal event, but even that memory was in the borderline range in comparison to controls. The other patients in the present study were also unimpaired in retrieving a detailed episodic memory from this time period. This correspondence in performance in the patients may relate to the "reminiscence bump," a phenomenon of disproportionately better retrieval of memories from adolescence to young adulthood in middle-aged and older adults (Rubin \& Schulkind, 1997). It may be that memory for these events, which are more vivid, personally relevant, and deeply encoded, is more protected from the effects of brain damage. H. M.'s teenage period memory had not been generated previously. Although this intact remote memory may be superficially interpreted as supporting SCT (Bayley et al., 2005), his autobiographical episodic recall was, in fact, profoundly impaired, with this event being the only temporally and spatially specific AM recalled from his entire life despite vigorous cueing.

Especially revealing is that, unlike $\mathrm{H}$. M. and K. C., a comparable profile to S. J. was reported in case A. D., who presented with bilateral lesions restricted to the fornix, which represents the major output from the hippocampus, and a small lesion to the basal forebrain (Gilboa et al., 2006). Follow-up investigation using an autobiographical recognition paradigm revealed that A. D.'s remote memory impairment was specific to episodic details of personal events and did not extend to memory for generic or semantic details of the same events (Gilboa et al., 2006). This study is key in its demonstration that autobiographical episodic memories can be selectively impaired following disruption to the extended hippocampal system (hippocampal formation, fornix, mammillary bodies, anterior thalamic nuclei) independent of other MTL or extra-MTL neocortical structures. This study also provides evidence that it is possible to obtain a severe and extensive retrograde amnesia for autobiographical events without damage to a distributed network of neocortical structures (Bright et al., 2006; Kopelman \& Kapur, 2001).

A separate set of MTL patients has been examined by Bayley et al. (2005), some with lesions restricted to the hippocampus and others with additional neocortical damage, using an adaptation of the autobiographical interview that was purported to be equivalent to the task used in the present study in detecting loss of episodic details. In those studies, conclusions of neocortical involvement in AM were based on a whole-brain volumetric analysis that divided the brain into the four lobes, the parahippocampal gyrus, the fusiform gyrus, and the insular cortex (see also Gold \& Squire, 2005). However, even finer regional parcellation and manual tracing in the present study did not show major extra-hippocampal MTL and neocortical loss in Patient S. J. greater than that exhibited by the other patients. If loss in neocortical 
regions damaged in S. J. contributed to his deficit, we would have expected a similar pattern of AM loss in Patient C. B., who had comparable damage, as well as additional deficits on neuropsychological tests of visuospatial function in relation to the parietal cortex, but this was not the case. Moreover, S. J.'s profound bilateral hippocampal volume loss was accompanied by less severe volume loss in left entorhinal and bilateral parahippocampal regions. It is possible that such damage may contribute to patterns of AM loss in MTL patients (e.g., Reed \& Squire, 1998). However, such an explanation is unlikely to account for the findings in the present study, where S. J.'s extra-hippocampal MTL volume loss was surpassed in each region in at least two other patients with less extensive episodic AM loss. In general, volumetric data must be considered in the context of the functional status of the brain regions in question if the data are to be interpretable. Moreover, quantitative analysis of local brain volumes does not rule out the possibility of a functional disconnection among a network of regions.

Differences between our results and those of Bayley et al. (2005) are likely due to a number of factors related to test administration and scoring procedures, despite the claim that their test is similar in sensitivity to the one used here. One main difference in administration is that Bayley et al.'s protocol yielded, on average, less than a third the number of details per memory in control participants, whether middle-aged or old, than does the current protocol under specific probing. Aging alone should have led to considerable differences in the ability to retrieve remote episodic memories (Levine et al., 2002; Piolino, Desgranges, Benali, \& Eustache, 2002). This difference may be explained by the fact that participants in the present study were asked to retrieve only one memory from each of five time periods, whereas Bayley et al. sampled 24 memories from the first third of life using Crovitz-like cue words as prompts (Crovitz \& Schiffman, 1974). A composite score derived from multiple memories is more reliable than one based on a single memory per time period. However, this does not apply to cases in which a patient has difficulty retrieving details from any time period. Moreover, the greater demand to retrieve a large number of remote memories may have resulted in a tradeoff in the form of generic descriptions of well-rehearsed events or in the exclusion of certain event details that would have otherwise been available to participants to recount. Squire and Bayley (2007) recently commented that patients with extensive remote memory loss, with the exception of V. C. described by Cipolotti et al. (2001) and possibly T. T. described by Maguire et al. (2006), had damage to extra-hippocampal structures. In all cases, the damage was minimal and comparable to that of the patients described in the present study, whose remote memory loss was relatively moderate in comparison to patients with large hippocampal lesions. Contrary to Squire and
Bayley, we believe that extensive hippocampal damage is likely to be responsible for the patients' severe memory loss.

The studies by Hepner, Mohamed, Fulham, and Miller (2007), Bright et al. (2006), and Kopelman et al. (2003), in contrast, only used a qualitative scoring procedure, and had similar difficulties to the studies by Bayley et al. (2005) in terms of the richness and depth of memories that were elicited given the greater number of memories sampled from fewer time periods. In the case of Bright et al., a modified version of the AMI was introduced that included more event topics from which to choose to aid memory retrieval, although it did not include additional probing after a memory was recalled freely. Even patients with restricted hippocampal lesions performed noticeably worse in retrieving personal events from the most remote and recent time periods, but not public event or semantic memories, although the variability in this group's data due to the small number of patients per group may account for the absence of statistical significance. Moreover, controls in this study seemed better able to retrieve remote than recent episodic memories, which is opposite to the pattern normally reported in healthy adults (Levine et al., 2002; Moscovitch et al., 2000). Hepner et al. (2007) used the standard AMI, together with a modified Crovitz cue word technique, to test a case with greater right than left MTL damage, and found a temporal gradient in AM similar to the pattern observed in Patient D. A., who also presents with partial (left) MTL sparing. Taking these issues into consideration, MTT still provides the best account of the findings with more rigorous testing when used in combination with detailed volumetric analysis.

\section{Conclusion}

The autobiographical interview, analyzed in the context of detailed volumetric data, helps to resolve discrepancies regarding hippocampal involvement in autobiographical re-experiencing. Although the cases described in the present study have MTL and posterior neocortical damage, the most parsimonious interpretation of the results is that extensive, bilateral hippocampal damage is to the most important factor contributing to severe, temporally extended retrograde memory impairment for episodic details. Small hippocampal lesions are not associated with severe retrograde amnesia even if accompanied by extensive, posterior neocortical damage. ${ }^{2}$ Technically speaking, we cannot assert with as much certainty, based on our data or on data from other human studies, whether extensive, bilateral hippocampal lesions are also sufficient to produce severe, temporally extended retrograde amnesia because some extra-hippocampal damage, however minute, is evident in every case. However, based on studies showing remote memory loss in rodents in which lesions are restricted to the hippocampus, and extrapolating from the few cases in humans, such as S. J. in the 
present study, as well as A. D., V. C., and T. T., whose extra-hippocampal lesions are small but whose hippocampal lesions are extensive, we believe that large, bilateral hippocampal lesions are also likely sufficient to produce severe, temporally extended retrograde amnesia. This suggests, consistent with MTT, that the hippocampus is needed to support autobiographical re-experiencing of remote and recent events (Moscovitch et al., 2006).

\section{Acknowledgments}

We thank S. J., R. G., C. B., and D. A. for their participation. We also thank Ariana Andrei, Sabitha Kanagasabai, Marina Mandic, and Irina Nica for assistance with the analyses and figures. Kate Frencham and Steven Jongenelis are thanked for collecting some of S. J.'s clinical data. This research was supported by grants from the Natural Sciences and Engineering Research Council to R. S. R. (Grant no. 312639), Canadian Institutes of Health Research to M. M. and R. S. R. (Grant no. MOP-135808) and B. L. (Grant nos. MGP-62963 and MOP-108540), and the National Institute of Child Health and Human Development to B. L. (Grant no. HD42385-01). J. F. K. acknowledges funding from the Raine Medical Research Foundation and the CSIRO Australian Imaging, Biomarker, and Lifestyle (AIBL) Flagship Study of Ageing. M. V. wishes to acknowledge the Office of Research and Development, Medical Research Service, Department of Veterans Affairs.

Reprint requests should be sent to R. Shayna Rosenbaum, Department of Psychology, York University, 4700 Keele St., Toronto, ON, Canada M3J 1P3, or via e-mail: shaynar@yorku.ca.

\section{Notes}

1. Unless indicated otherwise, the term MTL is used to refer to the hippocampus and related MTL cortical structures. The term neocortex is reserved for neocortical structures located outside of the MTL.

2. It should be noted that none of our patients had much damage to the retrosplenial cortex, where large lesions are associated with severe anterograde amnesia (Valenstein et al., 1987) and may produce comparable retrograde loss.

\section{REFERENCES}

Addis, D. R., Moscovitch, M., Crawley, A. P., \& McAndrews, M. P. (2004). Recollective qualities modulate hippocampal activation during autobiographical memory retrieval. Hippocampus, 14, 752-762.

Arndt, S., Cohen, G., Alliger, R. J., Swayze, V. W., II, \& Andreasen, N. C. (1991). Problems with ratio and proportion measures of imaged cerebral structures. Psychiatry Research, 40, 79-89.

Bayley, P. J., Gold, J. J., Hopkins, R. O., \& Squire, L. R. (2005). The neuroanatomy of remote memory. Neuron, 46 , 799-810.

Bayley, P. J., Hopkins, R. O., \& Squire, L. R. (2006). The fate of old memories after medial temporal lobe damage. Journal of Neuroscience, 26, 13311-13317.

Bright, P., Buckman, J., Fradera, A., Yoshimasu, H., Colchester, A. C., \& Kopelman, M. D. (2006). Retrograde amnesia in patients with hippocampal, medial temporal, temporal lobe, or frontal pathology. Learning and Memory, 13, 545-557.
Cabeza, R., \& St. Jacques, P. (2007). Functional neuroimaging of autobiographical memory. Trends in Cognitive Neurosciences, 11, 219-227.

Callen, D. J., Black, S. E., Gao, F., Caldwell, C. B., \& Szalai, J. P. (2001). Beyond the hippocampus: MRI volumetry confirms widespread limbic atrophy in AD. Neurology, 57, 1669-1674.

Cipolotti, L., Shallice, T., Chan, D., Fox, N., Scahill, R., Harrison, G., et al. (2001). Long-term retrograde amnesia... the crucial role of the hippocampus. Neuropsychologia, 39, 151-172.

Clark, R. E., Broadbent, N. J., \& Squire, L. R. (2005a). Hippocampus and remote spatial memory in rats. Hippocampus, 15, 260-272.

Clark, R. E., Broadbent, N. J., \& Squire, L. R. (2005b). Impaired remote spatial memory after hippocampal lesions despite extensive training beginning early in life. Hippocampus, 15 , 340-346.

Collins, D. L., \& Evans, A. C. (1997). ANIMAL: Validation and applications of non-linear registration based segmentation. International Journal of Pattern Recognition, 11, 1271-1294.

Crawford, J. R., \& Howell, D. C. (1998). Comparing an individual's test score against norms derived from small samples. The Clinical Neuropsychologist, 12, 482-486.

Crovitz, H. F., \& Schiffman, H. (1974). Frequency of episodic memories as a function of their age. Bulletin of the Psychonomic Society, 4, 517-518.

Dade, L. A., Gao, F. Q., Kovacevic, N., Roy, P., Rockel, C., O'Toole, C. M., et al. (2004). Semiautomatic brain region extraction: A method of parcellating brain regions from structural magnetic resonance images. Neuroimage, 22, 1492-1502.

Eustache, F., Piolino, P., Giffard, B., Viader, F., De La Sayette, V., Baron, J. C., et al. (2004). "In the course of time": A PET study of the cerebral substrates of autobiographical amnesia in Alzheimer's disease. Brain, 127, 1549-1560.

Feinstein, A., Roy, P., Lobaugh, N., Feinstein, K., O'Connor, P., \& Black, S. (2004). Structural brain abnormalities in multiple sclerosis patients with major depression. Neurology, 62, 586-590.

Fujii, T., Moscovitch, M., \& Nadel, L. (2000). Memory consolidation, retrograde amnesia, and the temporal lobe. In L. Cermak (Ed.), Handbook of neuropsychology (2nd ed., pp. 223-250). Amsterdam: Elsevier.

Gagnon, S., Foster, J. K., Turcotte, J., \& Jongenelis, S. (2004). Involvement of the hippocampus in implicit learning of supra-span sequences: The case of S.J. Cognitive Neuropsychology, 21, 867-882.

Gilboa, A., Ramirez, J., Köhler, S., Westmacott, R., Black, S. E., \& Moscovitch, M. (2005). Retrieval of autobiographical memory in Alzheimer's Disease: Relation to volumes of medial temporal lobe and other structures. Hippocampus, 15, 535-550.

Gilboa, A., Winocur, G., Grady, C. L., Hevenor, S. J., \& Moscovitch, M. (2004). Remembering our past: Functional neuroanatomy of recollection of recent and very remote personal events. Cerebral Cortex, 14, 1214-1225.

Gilboa, A., Winocur, G., Rosenbaum, R. S., Poreh, A., Gao, F., Black, S. E., et al. (2006). Hippocampal contributions to recollection in retrograde and anterograde memory: Evidence from a person with bilateral fornix and septal lesions. Hippocampus, 16, 966-980.

Gold, J. J., \& Squire, L. R. (2005). Quantifying medial temporal lobe damage in memory-impaired patients. Hippocampus, 15, 79-85.

Hepner, I. J., Mohamed, A., Fulham, M. J., \& Miller, L. A. (2007). Topographical, autobiographical and semantic 
memory in a patient with bilateral mesial temporal and retrosplenial infarction. Neurocase, 13, 97-114.

Insausti, R., Juottonen, K., Soininen, H., Insausti, A. M., Partanen, K., Vainio, P., et al. (1998). MR volumetric analysis of the human entorhinal, perirhinal, and temporopolar cortices. American Journal of Neuroradiology, 19, 659-667.

Kopelman, M. D., \& Kapur, N. (2001). The loss of episodic memories in retrograde amnesia: Single-case and group studies. Philosophical Transactions of the Royal Society of London, Series B, Biological Sciences, 356, 1409-1421.

Kopelman, M. D., Lasserson, D., Kingsley, D. R., Bello, F., Rush, C., Stanhope, N., et al. (2003). Retrograde amnesia and the volume of critical brain structures. Hippocampus, 13, 879-891.

Kopelman, M. D., Wilson, B. A., \& Baddeley, A. D. (1990). The autobiographical memory interview. Suffolk, England: Thames Valley Test Company.

Kovacevic, N., Henderson, J. T., Chan, E., Lifshitz, N., Bishop, J., Evans, A. C., et al. (2005). A three-dimensional MRI atlas of the mouse brain with estimates of the average and variability. Cerebral Cortex, 15, 639-645.

Kovacevic, N., Lobaugh, N. J., Bronskill, M. J., Levine, B., Feinstein, A., \& Black, S. E. (2002). A robust method for extraction and automatic segmentation of brain images. Neuroimage, 17, 1087-1100.

Levine, B. (2004). Autobiographical memory and the self in time: Brain lesion effects, functional neuroanatomy, and lifespan development. Brain and Cognition, 55, 54-68.

Levine, B., Svoboda, E., Hay, J. F., Winocur, G., \& Moscovitch, M. (2002). Aging and AM: Dissociating episodic from semantic retrieval. Psychology and Aging, 17, 677-689.

Maguire, E. A. (2001). Neuroimaging studies of autobiographical event memory. Philosophical Transactions of the Royal Society of London, Series B, Biological Sciences, 356, 1441-1451.

Maguire, E. A., Nannery, R., \& Spiers, H. J. (2006). Navigation around London by a taxi driver with bilateral hippocampal lesions. Brain, 129, 2894-2907.

Martin, S. J., de Hoz, L., \& Morris, R. G. (2005). Retrograde amnesia: Neither partial nor complete hippocampal lesions in rats result in preferential sparing of remote spatial memory, even after reminding. Neuropsychologia, 43, 609-624.

McKinnon, M. C., Black, S. E., Miller, B., Moscovitch, M., \& Levine, B. (2006). Autobiographical memory in semantic dementia: Implication for theories of limbic-neocortical interaction in remote memory. Neuropsychologia, 44, 2421-2429.

Milner, B., Squire, L. R., \& Kandel, E. R. (1998). Cognitive neuroscience and the study of memory. Neuron, 20, 445-468.

Moscovitch, M., Nadel, L., Winocur, G., Gilboa, A., \& Rosenbaum, R. S. (2006). The cognitive neuroscience of remote memory: A focus on functional neuroimaging. Current Opinion in Neurobiology, 16, 179-190.

Moscovitch, M., Rosenbaum, R. S., Gilboa, A., Addis, D. R., Westmacott, R., Grady, C. L., et al. (2005). Functional neuroanatomy of remote episodic (autobiographical), semantic and spatial memory in humans as determined by lesion and functional neuroimaging studies: A unified account based on Multiple Trace Theory. Journal of Anatomy, 207, 35-66.

Moscovitch, M., Yaschyshyn, T., Ziegler, M., \& Nadel, L. (2000). Remote episodic memory and retrograde amnesia: Was Endel Tulving right all along? In E. Tulving (Ed.), Memory, consciousness and the brain: The Tallinn Conference (pp. 331-345). Philadelphia, PA: Psychology Press/Taylor \& Francis.

Murray, E. A., \& Bussey, T. J. (2001). Consolidation and the medial temporal lobe revisited: Methodological considerations. Hippocampus, 11, 1-7.

Nadel, L., \& Moscovitch, M. (1997). Memory consolidation, retrograde amnesia and the hippocampal complex. Current Opinion in Neurobiology, 7, 217-227.

Nadel, L., \& Moscovitch, M. (2001). The hippocampal complex and long-term memory revisited. Trends in Cognitive Sciences, 5, 228-230.

Piolino, P., Desgranges, B., Benali, K., \& Eustache, F. (2002). Episodic and semantic remote autobiographical memory in aging. Memory, 10, 239-257.

Reed, J. M., \& Squire, L. R. (1998). Retrograde amnesia for facts and events: Findings from four new cases. Journal of Neuroscience, 18, 3943-3954.

Ribot, T. (1881). Les maladies de la memoire. Paris: Germer Baillare.

Rosenbaum, R. S., McKinnon, M., Levine, B., \& Moscovitch, M. (2004). Visual imagery deficits, impaired strategic retrieval, or memory loss: Disentangling the nature of an amnesic person's AM deficit. Neuropsychologia, 42, 1619-1635.

Rosenbaum, R. S., Winocur, G., \& Moscovitch, M. (2001) New views on old memories: Re-evaluating the role of the hippocampal complex. Behavioural Brain Research, 127, 183-197.

Rubin, D. C., \& Greenberg, D. L. (1998). Visual memory-deficit amnesia: A distinct amnesia presentation and etiology. Proceedings of the National Academy of Sciences, U.S.A., 95, 5413-5416.

Rubin, D. C., \& Schulkind, M. D. (1997). The distribution of autobiographical memories across the lifespan. Memory $\mathcal{E}$ Cognition, 25, 859-866.

Ryan, L., Nadel, L., Keil, K., Putnam, K., Schnyer, D., Trouard, T., et al. (2001). Hippocampal complex and retrieval of recent and very remote autobiographical memories: Evidence from functional magnetic resonance imaging in neurologically intact people. Hippocampus, 11, 707-714

Sanders, H. I., \& Warrington, E. K. (1971). Memory for remote events in amnesic patients. Brain, 94, 661-668.

Schnyer, D. M., Dobbins, I. G., Nicholls, L., Schacter, D. L., \& Verfaellie, M. (2006). Rapid response learning in amnesia: Delineating associative learning components in repetition priming. Neuropsychologia, 44, 140-149.

Scoville, W. B., \& Milner, B. (1957). Loss of recent memory after bilateral hippocampal lesions. Journal of Neurology, Neurosurgery, and Psychiatry, 20, 11-21.

Squire, L. R. (1992). Memory and the hippocampus: A synthesis from findings with rats, monkeys and humans. Psychological Review, 99, 195-231.

Squire, L. R., \& Bayley, P. J. (2007). The neuroscience of remote memory. Current Opinion in Neurobiology, 17, 185-196.

Squire, L. R., Cohen, N. J., \& Nadel, L. (1984). The medial temporal region and memory consolidation: A new hypothesis. In H. Weingartner \& E. Parker (Eds.), Memory consolidation (pp. 185-210). Hillsdale: Erlbaum.

Squire, L. R., Stark, C. E., \& Clark, R. E. (2004). The medial temporal lobe. Annual Review of Neuroscience, 27, 279-306.

Steinvorth, S., Levine, B., \& Corkin, S. (2005). Medial temporal lobe structures are needed to re-experience remote autobiographical memories: Evidence from H.M. and W.R. Neuropsychologia, 43, 479-496.

Svoboda, E., McKinnon, M. C., \& Levine, B. (2006). The functional neuroanatomy of autobiographical memory: A meta-analysis. Neuropsychologia, 44, 2189-2208. 
Talairach, J., \& Tournoux, P. (1988). Co-planar stereotaxic atlas of the buman brain. New York: Thieme.

Valenstein, E., Bowers, D., Verfaellie, M., Heilman, K. M., Day, A., \& Watson, R. T. (1987). Retrosplenial amnesia. Brain, 110, 1631-1646.

Viard, A., Piolino, P., Desgranges, B., Chetelat, G., Lebreton, K., Landeau, B., et al. (2007). Hippocampal activation for autobiographical memories over the entire lifetime in healthy aged subjects: An fMRI study. Cerebral Cortex, 17, $2453-2467$.

Viskontas, I. V., McAndrews, M. P., \& Moscovitch, M. (2000). Remote episodic memory deficits in patients with unilateral temporal lobe epilepsy and excisions. Journal of Neuroscience, 20, 5853-5857.

Wechsler, D. (1987). Wechsler memory scale-Revised. San Antonio, TX: Psychological Corporation.

Winocur, G., Moscovitch, M., Caruana, D. A., \& Binns, M. A. (2005a). Retrograde amnesia in rats with lesions to the hippocampus on a test of spatial memory. Neuropsychologia, 43, 1580-1590.

Winocur, G., Moscovitch, M., Fogel, S., Rosenbaum, R. S., \& Sekeres, M. (2005b). Preserved spatial memory after hippocampal lesions: Effects of extensive experience in a complex environment. Nature Neuroscience, 8 , 273-275.

Woods, R. P., Grafton, S. T., Holmes, C. J., Cherry, S. R., \& Mazziotta, J. C. (1998). Automated image registration: I. General methods and intrasubject, intramodality validation. Journal of Computer Assisted Tomography, 22, 139-152.

Woods, R. P., Grafton, S. T., Watson, J. D., Sicotte, N. L., \& Mazziotta, J. C. (1998). Automated image registration: II. Intersubject validation of linear and nonlinear models. Journal of Computer Assisted Tomography, 22, 153-165.

Yonelinas, A. P., Kroll, N. E., Quamme, J. R., Lazzara, M. M., Sauve, M. J., Widaman, K. F., et al. (2002). Effects of extensive temporal lobe damage or mild hypoxia on recollection and familiarity. Nature Neuroscience, 5, 1236-1241.

Zola-Morgan, S., Squire, L. R., \& Amaral, D. G. (1986). Human amnesia and the medial temporal region: Enduring memory impairment following a bilateral lesion limited to field CA1 of the hippocampus. Journal of Neuroscience, 6, 2950-2967. 